\title{
Dic Medanif
}

\section{in sent \\ Midht-(Euklidirchen Maumformen.}

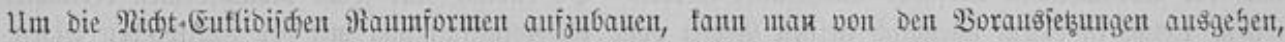
weldje Euflib implicite Durd) Die erjten Definitionen madt; man gelangt ban, wie id in Bordfarot's Jour.

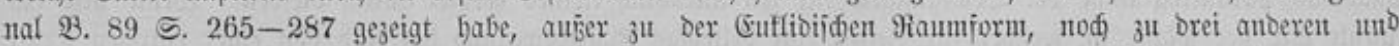

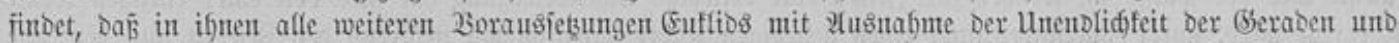

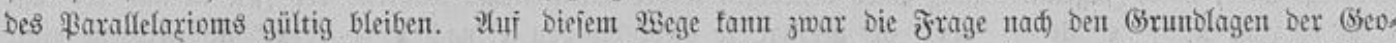
metrie nidjt principiell gelöjt werben, Dagegen füfjrt berfelbe gan melde mit unjerer (Exjabrung vereinbar jint.

Die auf biejem wege erlangten Raumformen fimmen in einem untendidg fleinen (5ebiet mit ber Euflibijđen überein. Un baher bie in ihnen bejtehentoen (Bejese ber Medfanif ju erioridjen, fann man Davon

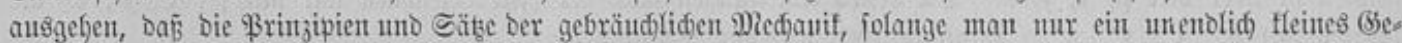

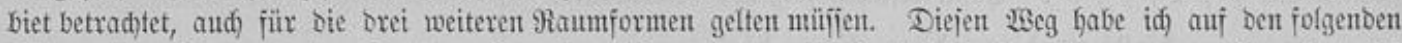
Seiten eingeid)lagent unto aujier ben allgemeinen formeln ber Mledanif biejenigen Bervegungent betrad)tet, bei

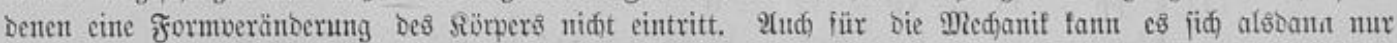

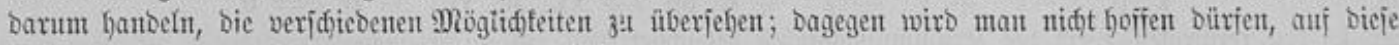
ISeije Die Jrage nad) ben Frinzipien ju erlebigen.

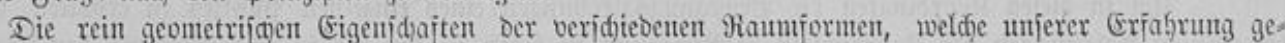

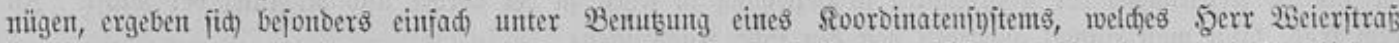

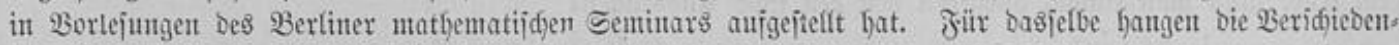

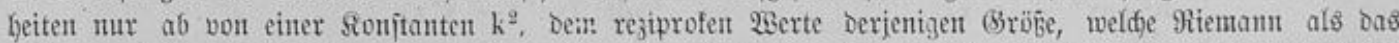

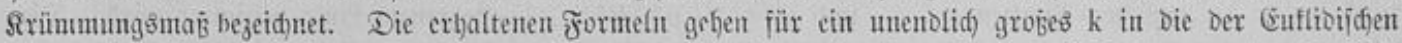
(Seometric bei redftwinfligen foorsinaten über, uno für $k=1$ tritt cine enge Fejiefung ju ber Ephärif

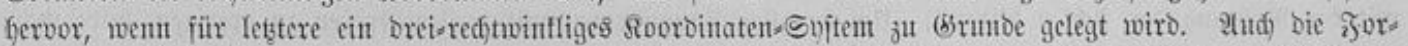

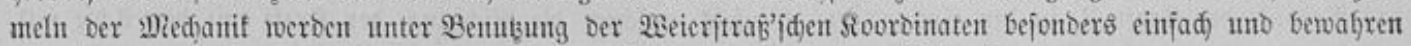

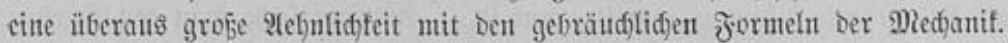

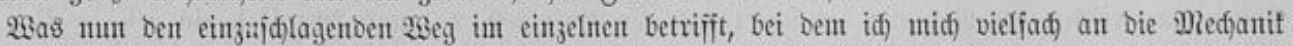

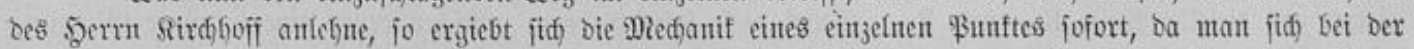

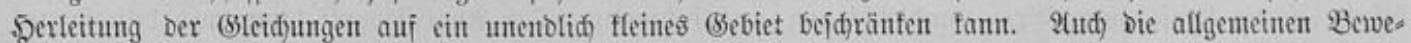

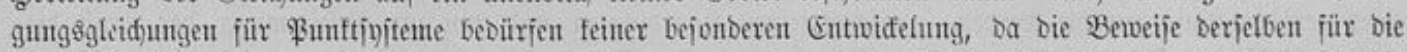

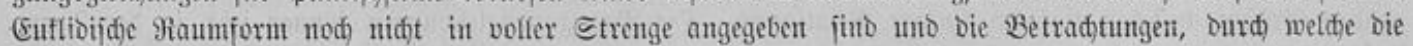

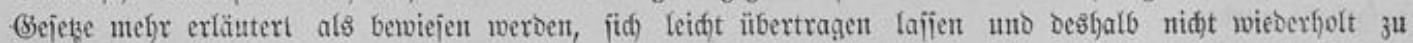
werben brauden. Die Stellung meiner Unterjud)ung ju vervanten früfyeren थrbeiten glaube id nidgt näber

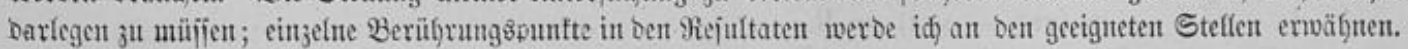




\section{Bewegunt eities freien \$unttes.}

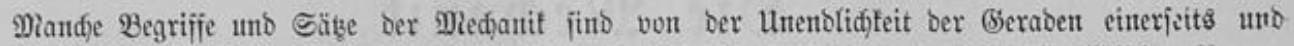

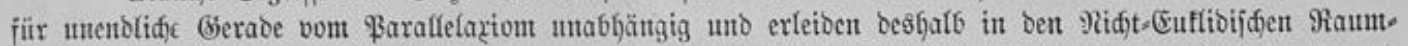

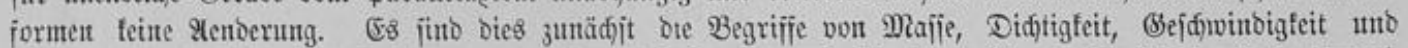

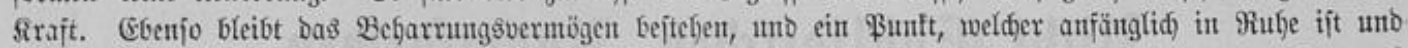

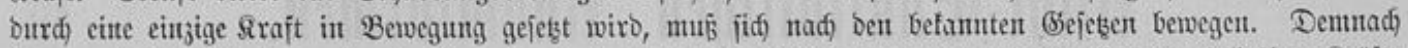
fant aud bie (Eintheit ber Sirajt affgemein in ber befaunten 2 seije befiniiert unt bie Miejiung beliebiger Sräfte

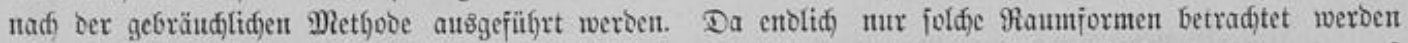

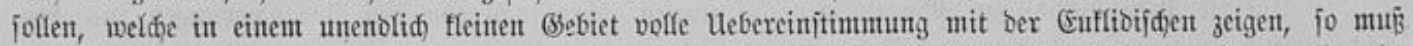

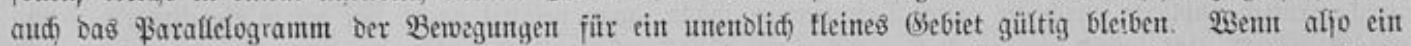
Bunft mit ber Maije $m$, weld)er zur 3eit $t$ cine beliebige Eigenbenvegung fat uno von einer einjigen Strajt

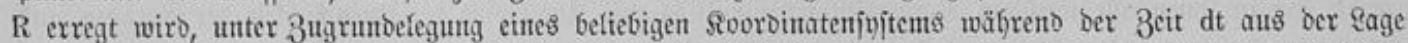
$x_{6}$ in bie Sage $x_{6}+x_{6}^{\prime} d t+1 / 2 x_{6}^{\prime \prime} d t^{2}$ gelangt, fo giebt biz von $x_{6}$ nad $x_{6}+1 / 2 x_{6}^{\prime \prime} d t^{2}$ gezogene Sintic

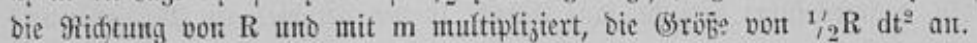

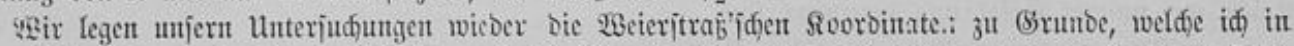

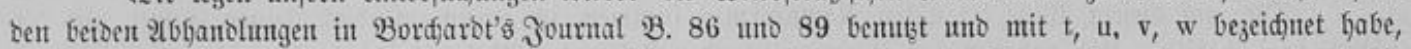
nemten fie aber hier $p, x, y, z$. Die erjten 2 (bleitungen nad) ber Beit bejeidnen wir mit $p^{\prime}, x^{\prime}, y^{\prime}, z^{\prime}$, bie jweiten mit $\mathrm{p}^{\prime \prime}, \mathrm{x}^{\prime \prime}, \mathrm{y}^{\prime \prime}, \mathrm{z}^{\prime \prime}$. Dann bejteben jolgenbe Relationen:

$$
\begin{aligned}
& \mathrm{k}^{2} \mathrm{p}^{2}+\mathrm{x}^{2}+\mathrm{y}^{2}+\mathrm{z}^{2}=\mathrm{k}^{2} \\
& \text { (1) } \mathrm{k}^{2} \mathrm{pp}^{\prime}+\mathrm{xx}^{\prime}+\mathrm{yy}^{\prime}+\mathrm{zz}^{\prime}=0 \\
& \mathrm{k}^{2} \mathrm{p}^{\prime 2}+\mathrm{x}^{\prime 2}+\mathrm{y}^{\prime 2}+\mathrm{z}^{\prime 2}+\mathrm{k}^{2} \mathrm{pp} \mathrm{p}^{\prime \prime}+\mathrm{xx}^{\prime \prime}+\mathrm{yy}^{\prime \prime}+z z^{\prime \prime}=0,
\end{aligned}
$$

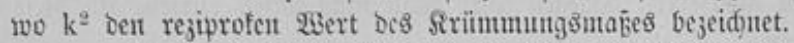

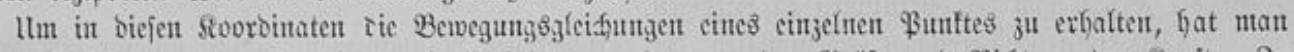

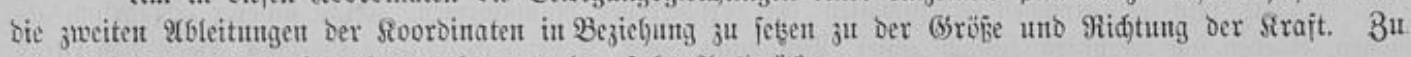

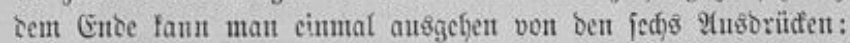

$$
1 / 2 d t^{2}\left(p x^{\prime \prime}-p x\right), \ldots . . .{ }^{1 / 2} d t^{2}\left(y z^{\prime \prime}-y^{\prime \prime} z\right), \ldots . . . .
$$

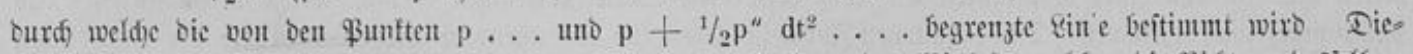

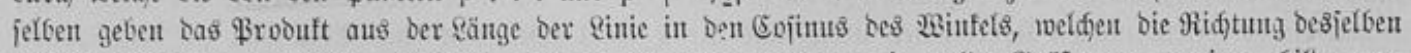

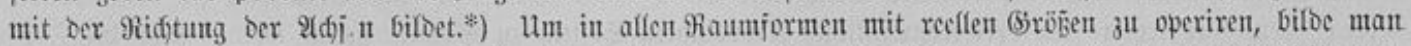

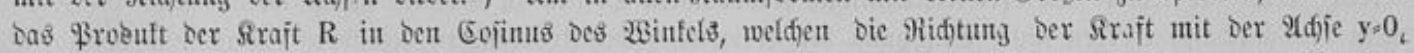

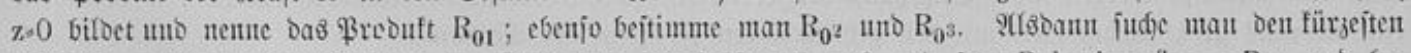

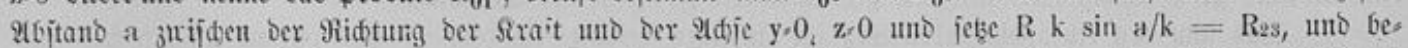

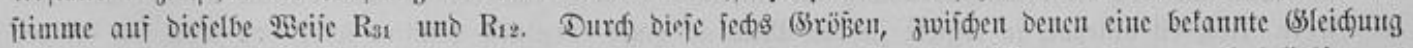

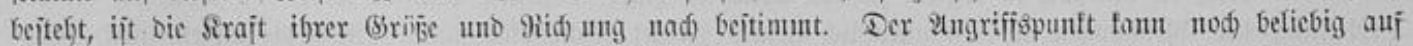

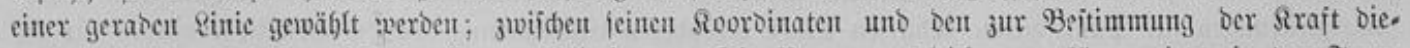

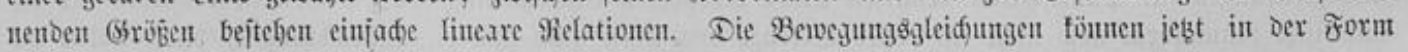
aufgejtelit werben:

$$
\text { (2) } \mathrm{m}\left(\mathrm{px}^{\prime \prime}-\mathrm{p}^{\prime \prime} \mathrm{x}\right)=\mathrm{R}_{01} \text {....... . . . . . . . . }
$$

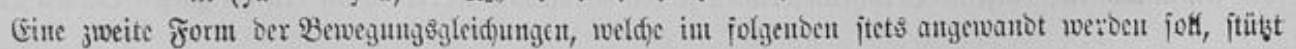

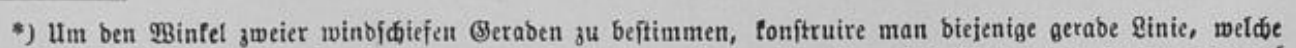
auf beiben fenfredt fteht nub lege burd bieje unb je eine ber gegebenen Seraben eine Ebene; ber Reigungşwintel biejer beiben Ebenen ift ber gejudte $2 B i n t d$. 


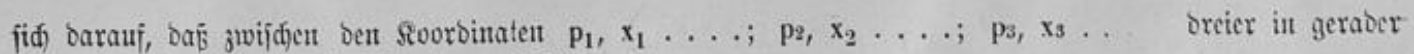
Sinte liegenoen Funfte die Relationen bejtehen:

$$
\begin{aligned}
& \alpha p_{1}+\beta p_{2}+\gamma p_{s}=0, \alpha x_{1}+\beta x_{2}+\gamma x_{3}=0 \\
& \alpha y_{1}+\beta y_{2}+\gamma y_{3}=0, c z_{1}+\beta z_{2}+\gamma z_{3}=0
\end{aligned}
$$

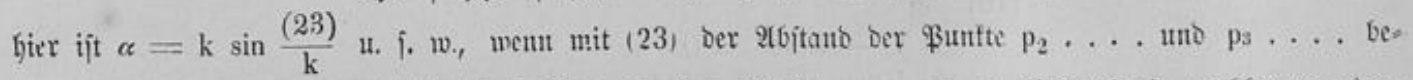

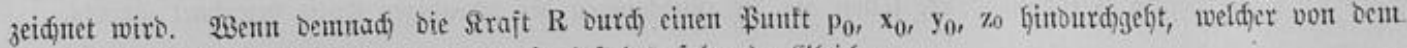
bewegten \$sunfte bie Entfernung o gat, jo beiteben folgente (Sícid)ungen

$$
\begin{aligned}
\mathrm{m} \mathrm{p}^{\prime \prime} & =\frac{\mathrm{R} \mathrm{p}_{0}}{\mathrm{k} \sin \mathrm{e} / \mathrm{k}}-\mathrm{L} \mathrm{p} \\
\mathrm{m} \mathrm{x}^{\prime \prime} & =\frac{\mathrm{R} \mathrm{x}_{0}}{\mathrm{k} \sin e / \mathrm{k}}-\mathrm{L} \mathrm{x} \\
\mathrm{m} \mathrm{y}^{\prime \prime} & =\frac{\mathrm{R} \mathrm{y}_{0}}{\mathrm{k} \sin e / \mathrm{k}}-\mathrm{L} \mathrm{y} \\
\mathrm{m} \mathrm{z}^{\prime \prime} & =\frac{\mathrm{B} \mathrm{z}_{0}}{\mathrm{k} \sin e / \mathrm{k}}-\mathrm{L} z .
\end{aligned}
$$

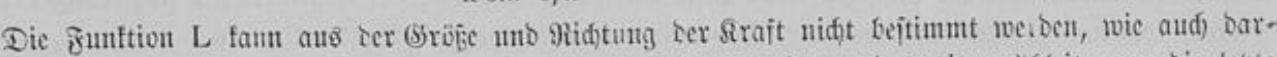

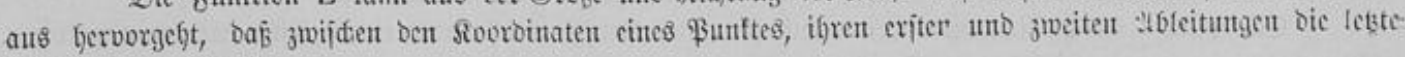
(Śleid)ung (1) bejtef)t.

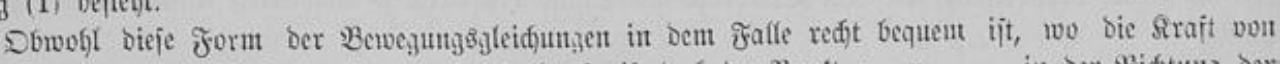

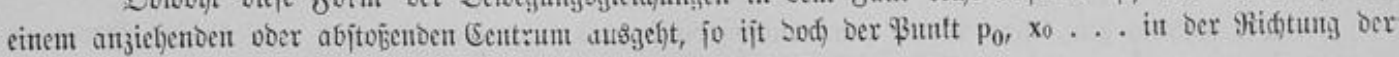

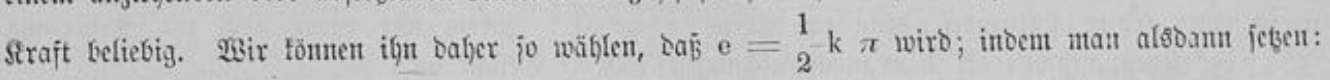

$$
R \mathrm{p}_{0}=\mathrm{k} P, \mathrm{R} \mathrm{x}_{0}=\mathrm{k} X, \mathrm{R} \mathrm{y}_{0}=\mathrm{k} \mathrm{Y}, \mathrm{R} \mathrm{z}_{0}=\mathrm{k} \mathrm{Z} \text {, }
$$

erjafiuten bie Benvegungsgleidjungen in Det form:

$$
\text { (4) } \begin{aligned}
\mathrm{m} \mathrm{p}^{\prime \prime} & =\mathrm{P}-\mathrm{S} \mathrm{p} \\
\mathrm{m} \mathrm{y}^{\prime \prime} & =\mathrm{X}-\mathrm{S} \mathrm{x}-\mathrm{S} \mathrm{y} \\
\mathrm{m} \mathrm{z}^{\prime \prime} & =\mathrm{Z}-\mathrm{S} \mathrm{z}
\end{aligned}
$$

wo jwijden $P, X \ldots$ unt $p, x \ldots$ dic (Sleidung bejteht:

$$
\text { (5) } k^{2} P p+X x+Y y+Z z=0
$$

Die (sröbe ber Sraft $R$ erg̣iebt jid) ous ber (sleid)ung:

$$
\text { (6) } \mathrm{R}^{2}=\mathrm{k}^{2} \mathrm{P}^{2}+\mathrm{X}^{2}+\mathrm{Y}^{2}+\mathrm{Z}^{2} \text {. }
$$

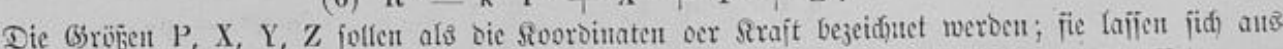

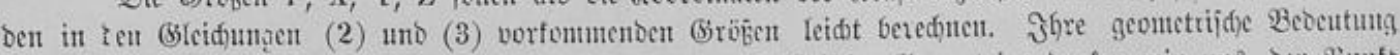

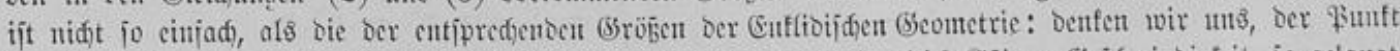

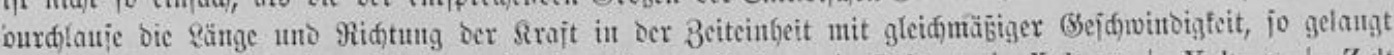
er in ber Beit dt nad) einem \$unfte, ecjien Soorbinaten find: $\mathrm{p}+\mathrm{Pdt}, \mathrm{x}+\mathrm{X}$ dt, $\mathrm{y}+\mathrm{Y} \mathrm{dt}, \mathrm{z}+\mathrm{Z}$ dt

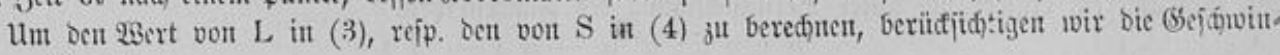
Digfeit v. Nun iit

\section{Setsen wir}

$$
\text { (7) } \mathrm{v}^{2}=\mathrm{k}^{2} \mathrm{p}^{\prime 2}+\mathrm{x}^{\prime 2}+\mathrm{y}^{\prime 2}+\mathrm{z}^{\prime 2} \text {. }
$$

$$
\text { (8) } 1 / 2 \mathrm{~m} \mathrm{v} \mathrm{v}^{3}=\mathrm{T} \text {, }
$$

fo engeben fid) unter Beriaffidftigung ber (s)cidungen (1) aus (3).

$$
\frac{d T}{d t}=\frac{R}{k \sin e / k}\left(k^{2} P_{0} p^{\prime}+x_{0} x^{\prime}+y_{0} y^{\prime}+z_{0} z^{\prime}\right)
$$


uno aแร (4):

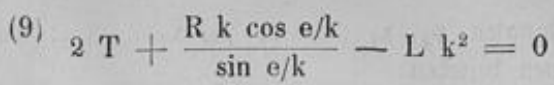

$$
\text { (10) } \frac{\mathrm{d} T}{\mathrm{dt}}=\mathrm{k}^{2} \mathrm{P} \mathrm{p}^{\prime}+\mathrm{X} \mathrm{x}^{\prime}+\mathrm{Y} \mathrm{y}^{\prime}+\mathrm{Z} \mathrm{z}^{\prime}
$$

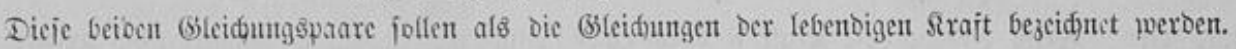

\section{Beipiel $\mathfrak{B l a n e t e n b e r e g u n g . ~}$}

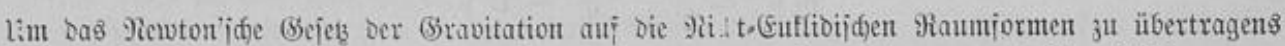

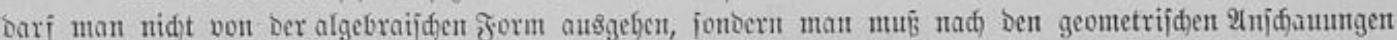
fragen, weldje bemijelben 3 (Srumbe licyen. Tenft man fid um ben anjiebentoen \$unft als (Eentrum meljrere

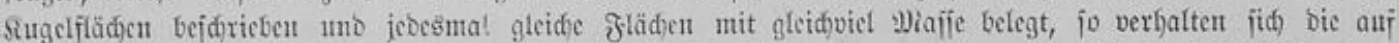

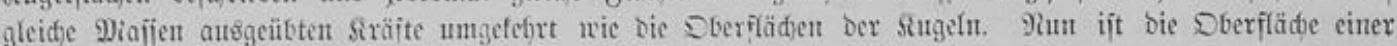
Sugel, beren Fabius gleid) $\mathrm{r}$ ijt, gleid) $4 \pi \mathrm{k}^{2} \sin ^{2} \mathrm{r} / \mathrm{k}$. Jntom wir Dafjer bie Sonne als unverveglid, ans

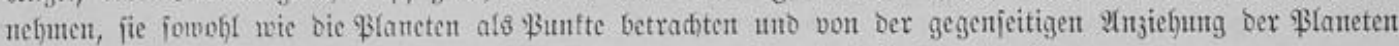

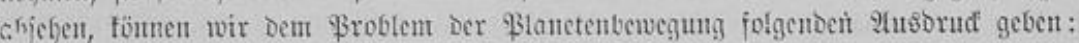

(Ein \$unft benege fid) unter beut Einfuije ciner firajt, weldfe nad) cinem feiten (Eentrum geridetet

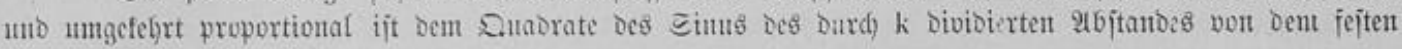
\$suntte

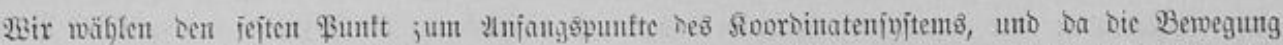

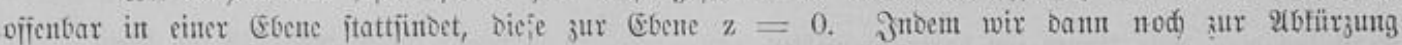
$x^{2}+y^{2}=q^{2}$ icken, nefhmen bic Bewegumgsgleid)utgen (3) Dic Bejtalt ant:

$$
\begin{aligned}
& p^{\prime \prime}=\frac{\mu}{4^{3}}-p L \\
& x^{\prime \prime}=-11 . \\
& y^{\prime \prime}=-y_{1} 1 .
\end{aligned}
$$

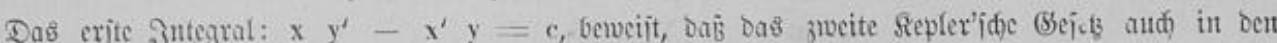

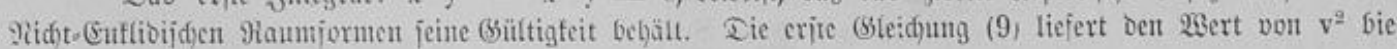

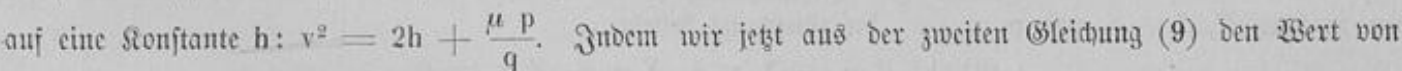
L unb bann aus Der (Sleidung $k^{2} p^{2}+q^{2}=k^{2}$ uno ifren beiben erịten Fbleitunger: Den WGert von $q^{\prime \prime}$ berednen, gelangen wir zu Den (Slcidungen:

$$
\begin{aligned}
& q x^{\prime \prime}-q^{\prime \prime} x=-\frac{c^{2} x}{q 3}=\frac{c}{\mu}\left(p x^{\prime \prime}-p^{\prime \prime} x\right) \\
& q y^{\prime \prime}-q^{\prime \prime} y=-\frac{c^{2} y}{q^{3}}=\frac{c^{2}}{\mu}\left(p y^{\prime \prime}-p^{\prime \prime} y\right) .
\end{aligned}
$$

Sndem wir bieje beiben (Sleid)ungen integrieren, bie erite mi: $\mathrm{y}$, bic jweite nit $\mathrm{x}$ multipitzieren

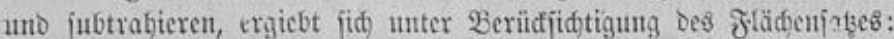

$$
\mu \mathrm{c} q=\mathrm{c}^{3} \mathrm{p}-\alpha \mathrm{y}+\beta \mathrm{x}
$$

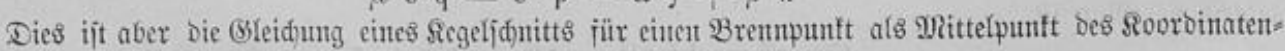

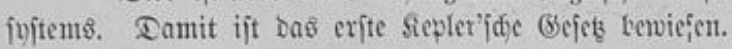

\section{Beifpicl. Wurfbeneaung.}

(Ein \$untt bewege jid) unter Dem (Einflujie ciner fonjtanten \&rajt, weldfe auf einer fejtent (Ebene

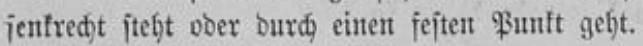


Da eine jolde firait it ber Natur faum vorfommen birite, jo genïge es, barauf aujmerfiam jut

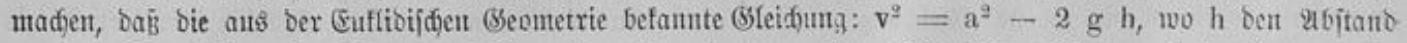

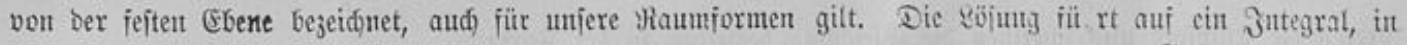

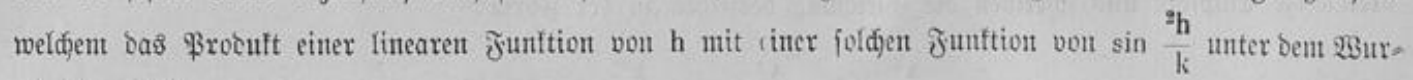
zelseidjeit iteljt.

\section{§. 2}

\section{Bemequı!n nuf eiller จึlüd)e.}

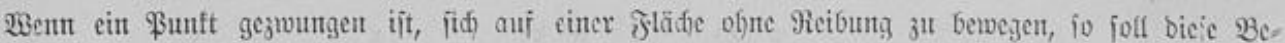

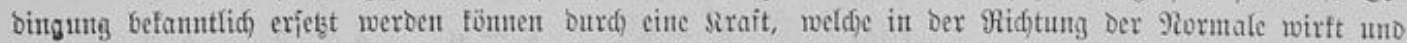

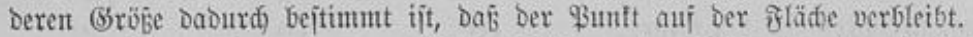

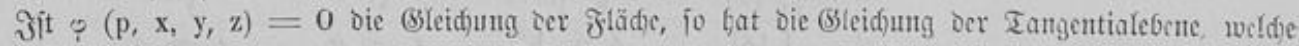

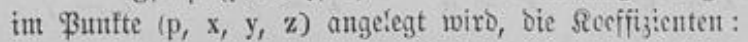

$$
\begin{aligned}
& \frac{1}{k^{2}} \frac{d p}{d p}-p\left(p \frac{d \varphi}{d p}+x \frac{d}{d} i+y \frac{d \varphi}{d y}+z \frac{d \varphi}{p z}\right) \\
& \frac{d \varphi}{d x}-x\left(p \frac{d \varphi}{d p}+x \frac{d \varphi}{d x}+y \frac{d \varphi}{d y}+z \frac{d \varphi}{d z}\right) \\
& \frac{d \varphi}{d y}-y\left(p \frac{d \varphi}{d p}+x \frac{d \varphi}{d x}+y \frac{d \varphi}{d z}+z \frac{d \varphi}{d z}\right) \\
& \frac{d \varphi}{d z}-z\left(p \frac{d \varphi}{d h}+x \frac{d}{d x}+y \frac{d \varphi}{d z}+z \frac{d \varphi}{d z}\right)
\end{aligned}
$$

Somit liegt aui ber Normale $0 . \&$ Buftes ( $\mathrm{p}, \mathrm{x}, \mathrm{y}, \mathrm{z}$ ) ein reelfer ober imaginärer \$untt, Defïen

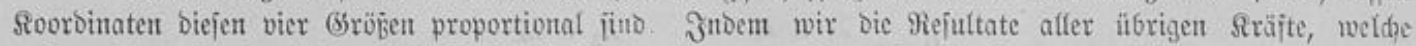

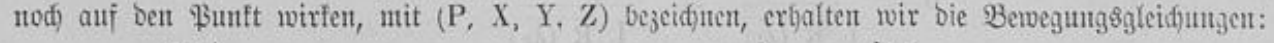

$$
\begin{aligned}
& m p^{\mu}=P-S p+\frac{l}{k^{2}} M \frac{d \varphi}{d p} \\
& m x^{\prime \prime}=x-s x+M \frac{d \varphi}{d x} \\
& m y^{\prime \prime}=Y-S y+M \frac{d \varphi}{d y} \\
& \mathrm{~m} \mathrm{z}^{\prime \prime}=\mathrm{Z}-\mathrm{S} \mathrm{z}+\mathrm{M} \frac{\mathrm{d} \varphi}{\mathrm{d} z}
\end{aligned}
$$

Die erite (steidung (10) Der lebendigen firait bleibt aud) in Dicjent Jalfe gültig; Die jweite änbert

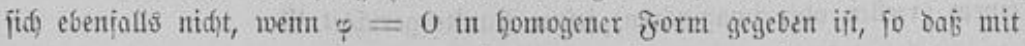

$$
\varphi \text { aud } p \frac{d \varphi}{d p}+x \frac{d \varphi}{d x}+y \frac{d \varphi}{d y}+z \frac{d \varphi}{d z} \text { veridivimbet. }
$$

\section{Beijpiel. Sï̈rzeite Rinten auf eitrer Flärhe.}

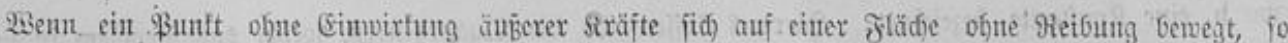

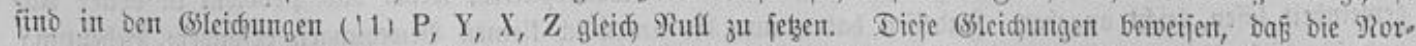

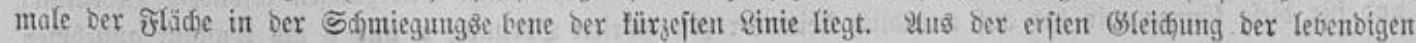




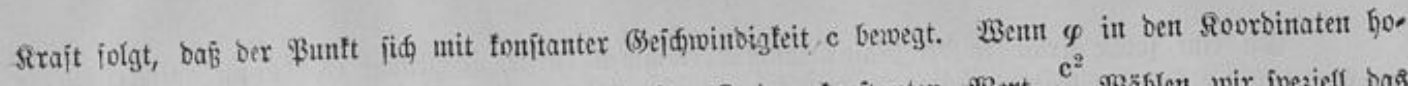

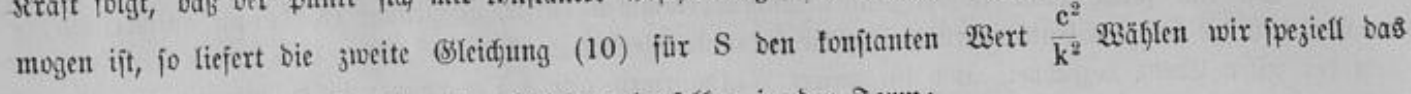

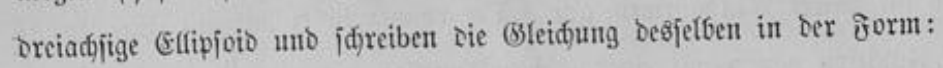

$$
\frac{\mathrm{x}^{2}}{\alpha}+\frac{\mathrm{y}^{2}}{\beta}+\frac{\mathrm{z}^{2}}{\gamma}-\mathrm{p}^{2}=0
$$

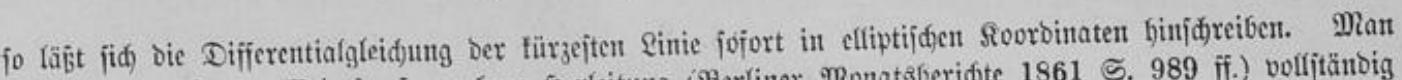

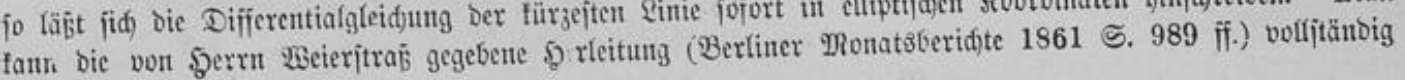
übertragen, weun man jeş̧ :

$$
\begin{aligned}
& \mathrm{f}(\lambda)=(\lambda-\alpha)(\lambda-\beta)(\lambda-\gamma) \frac{\lambda+\mathrm{k}^{2}}{\mathrm{k}^{2}} \\
& \frac{\varphi_{(\lambda)}}{\mathrm{f}(\lambda)}=\frac{\mathrm{p}^{2} \mathrm{k}^{2}}{\lambda+\mathrm{k}^{2}}+\frac{\mathrm{x}^{2}}{\lambda-\alpha}+\frac{\mathrm{y}^{2}}{\lambda-\beta}+\frac{\mathrm{z}^{ \pm}}{\lambda-\gamma} \\
& \frac{\varphi_{1}(\lambda)}{\mathrm{f}(\lambda)}=\frac{\mathrm{k}^{2} \mathrm{p}^{\prime 2}}{\lambda+\mathrm{k}^{2}}+\frac{\mathrm{x}^{\prime 2}}{\lambda-\alpha}+\frac{\mathrm{y}^{\prime 2}}{\lambda-\beta}+\frac{\mathrm{z}^{\prime 2}}{\lambda-\gamma}
\end{aligned}
$$

Ulsbann crgiebt jith auj Dem bort angegebenen Wege:

$$
\begin{aligned}
& \left(\frac{1}{2} \frac{d \varphi(\lambda)}{d \cdot t}\right)^{2}-\varphi(\lambda) \varphi(\lambda)= \\
& -\frac{c^{2}}{k^{2}}\left(\lambda+k^{2}\right)(\lambda-\alpha)(\lambda-\beta)(\lambda \quad \gamma) \lambda(\lambda-\delta)
\end{aligned}
$$

intbem man bie linte Scite gleid, $\mathrm{c}^{2} \mathrm{R}(\lambda)$ jeçt uno ntit $\lambda_{1}$ uno $\lambda_{2}$ biejen.gen $23 e r t e$ bejeidnet, weldje autger Null ber (sleid)ung $\varphi(\lambda)=0$ genüjen, ergeben fï) als (Sfeid)ungen ber fürzeiten Sinte:

$$
\begin{aligned}
& 0=\frac{\lambda_{1} \mathrm{~d} \lambda_{1}}{\sqrt{\mathrm{R}\left(\lambda_{1}\right)}}+\frac{\lambda_{2} \mathrm{~d} \lambda_{2}}{\sqrt{\mathrm{R}\left(\lambda_{2}\right)}} \\
& \mathrm{cdt}=\frac{\lambda_{1}{ }^{2} \mathrm{~d} \lambda_{1}}{\sqrt{\mathrm{R}\left(\lambda_{1}\right)}}+\frac{\lambda_{2}{ }^{2} \mathrm{~d} \lambda_{2}}{V \overline{\mathrm{R}\left(\lambda_{2}\right)}}
\end{aligned}
$$

\section{Beifpiel. Das Bendel.}

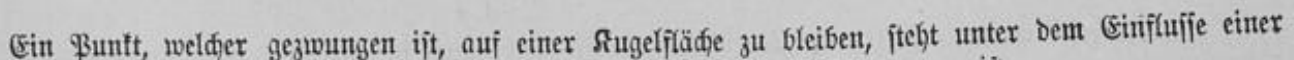

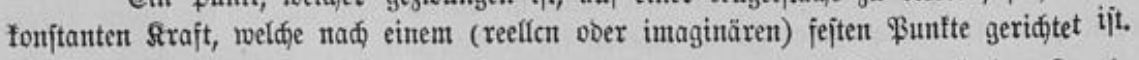

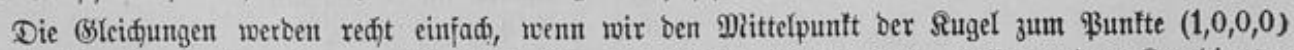

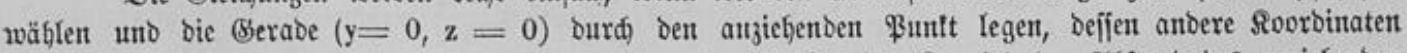

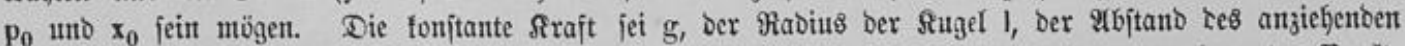

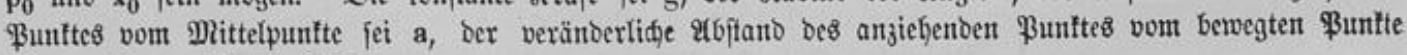
jei e. Dann gilt bie (Sleidung:

$$
\mathrm{v}^{2}=2 \mathrm{~g}(\mathrm{~h}-\mathrm{e}),
$$

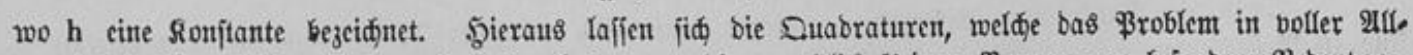

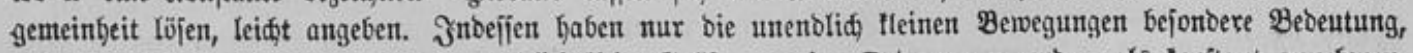

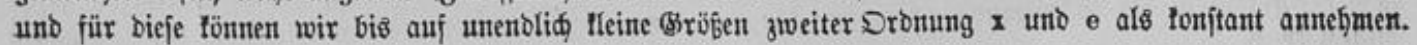




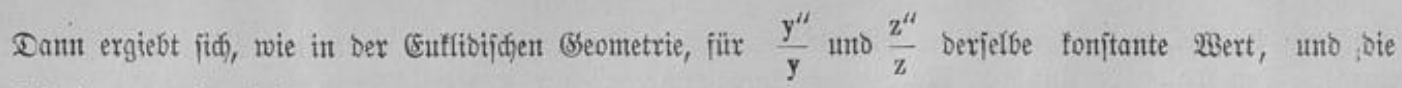
(d)wintgungşzeit wirb

$$
=2 \pi \sqrt{\frac{\mathrm{k} \sin \frac{\mathrm{l}}{\mathrm{k}}}{\mathrm{g}} \frac{\sin \frac{\mathrm{a}-1}{\mathrm{k}}}{\sin \frac{\mathrm{a}}{\mathrm{k}}}}
$$

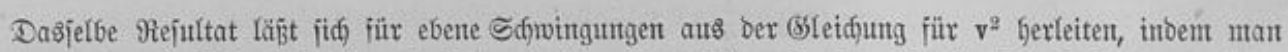

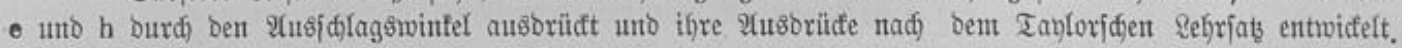

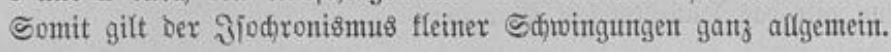

\section{§. 3 .}

\section{Die affgemteiten Bemegutgğgleid)unget.}

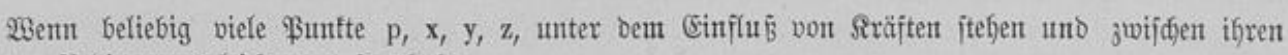
Soorbinaten Bebirgung gleidungen jtatthaben:

$$
\text { (12) } \varphi=0, \psi=0, \ldots
$$

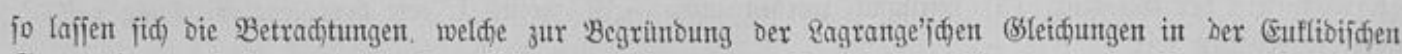

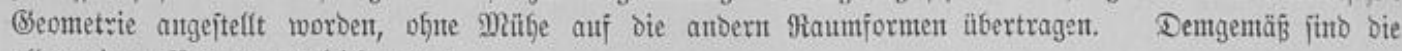
allgemeinten Bewegunge̊gleidjungen:

$$
\begin{aligned}
& \mathrm{m}_{\imath} \mathrm{P}_{\imath}{ }^{\prime \prime}=\mathrm{P}_{\imath}+\mathrm{S}_{\imath} \mathrm{p}_{\imath}+\frac{1}{\mathrm{k}_{2}} \mathrm{M} \frac{\partial \varphi}{\partial \mathrm{p}_{\imath}}+\frac{1}{\mathrm{k}_{2}} \mathrm{~N} \frac{\partial \psi}{\partial \mathrm{p} \iota}+\ldots \\
& \mathrm{m}^{\prime} \mathrm{x}_{i}{ }^{\prime \prime}=\mathrm{X}_{i}+\mathrm{S}_{i} \mathrm{x}_{i}+\mathrm{M} \frac{\partial \varphi}{\partial \mathrm{x}_{i}}+\mathrm{N} \frac{\partial \psi}{\partial \mathrm{z} i}+\ldots
\end{aligned}
$$

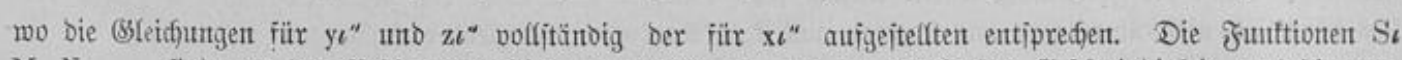

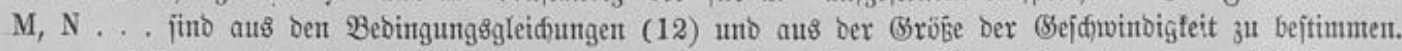

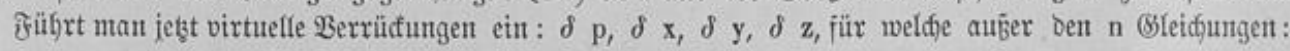

$$
\mathrm{k}^{2} \mathrm{p}_{i} \delta \mathrm{p}_{\mathrm{i}}+\mathrm{x}_{i} \delta \mathrm{x}_{\mathrm{i}}+\mathrm{y}_{\mathrm{i}} \delta \mathrm{y}_{\mathrm{i}}+\mathrm{z}_{\mathrm{i}} \delta \mathrm{z}_{\mathrm{i}}=0
$$

nod) bie weitern (Bleidungen bejtehen:

$$
\text { (14) } \Sigma\left(\frac{\partial \varphi}{\partial \mathrm{p}} \delta \mathrm{p}+\frac{\partial \varphi}{\partial \mathrm{x}} \delta \mathrm{x}+\frac{\partial \varphi}{\partial \mathrm{y}} \delta \mathrm{y}+\frac{\partial \varphi}{\partial \mathrm{z}} \delta \mathrm{z}\right)=0
$$

io erfält man jojort Das̊ b'2lembert'iđe ßrincip in ber form:

$$
\begin{gathered}
\Sigma\left(\mathrm{k}^{2}\left(\mathrm{~m} \mathrm{p}^{\prime \prime}-\mathrm{P}\right) \delta \mathrm{p}+\left(\mathrm{m} \mathrm{x}^{\prime \prime}-\mathrm{X}\right) \delta \mathrm{x}+\left(\mathrm{m} \mathrm{y}^{\prime \prime}-\mathrm{Y}\right) \delta \mathrm{y}\right. \\
\left.+\left(\mathrm{m} \mathrm{z}^{\prime \prime}-\mathrm{Z}\right) \delta \mathrm{z}\right)=0,
\end{gathered}
$$

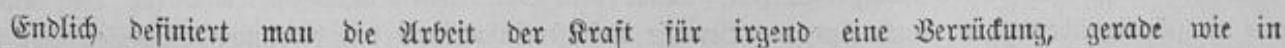
Der Euflibijden Geometrie, als bas \$robuft aus ber Sraft unb ber Berrüfung in ben Gofinus bes ein- 


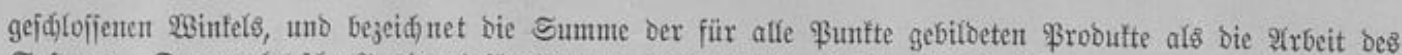

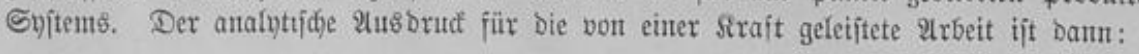

$$
\text { (16) } \mathrm{k}^{2} \mathrm{P} \delta \mathrm{p}+\mathrm{X} \delta \mathrm{x}+\mathrm{Y} \delta \mathrm{y}+\mathrm{Z} \delta \mathrm{z} \text {, }
$$

uno bie Bejamtarbeit $U^{\prime}$ wiro curd) eine über alfe ßunfte aus̊gebegnte Summation erfjaltent. Ebenio bes

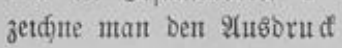

$$
\text { (17) } \frac{1}{2} \quad \mathrm{sm} \mathrm{v}^{2}=\mathrm{T}
$$

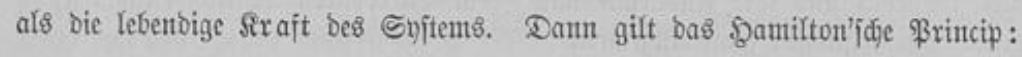

(18) $\int\left(\delta \mathrm{T}+\mathrm{U}^{\prime}\right) \mathrm{dt}=0$

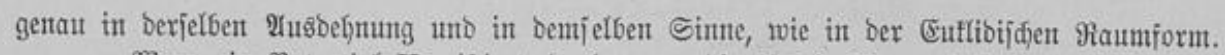

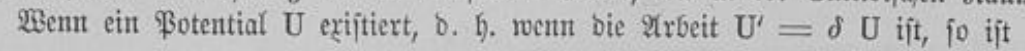

$$
\text { (19) } \begin{aligned}
\mathrm{P} & =\frac{1}{\mathrm{k}^{2}} \frac{\partial \mathrm{U}}{\partial \mathrm{p}}-\mathrm{E}_{\mathrm{p}, \mathrm{X}}=\frac{\partial \mathrm{U}}{\partial \mathrm{x}}-\mathrm{Ex}, \\
\mathrm{Y} & =\frac{\partial \mathrm{U}}{\partial \mathrm{g}}-\mathrm{E} \mathrm{y}, \mathrm{Z}=\frac{\partial \mathrm{U}}{\partial \mathrm{z}}-\mathrm{E}_{\mathrm{z}}
\end{aligned}
$$

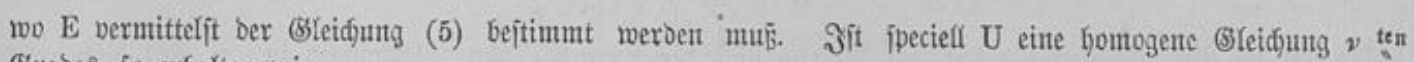
(6rabes, jo erfalten mir

$$
\text { (20) } \mathrm{k}^{2} \mathrm{E}=v \mathrm{U}
$$

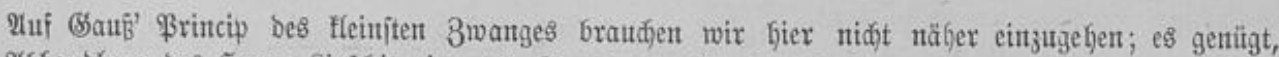

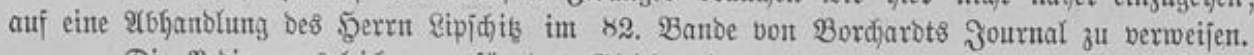

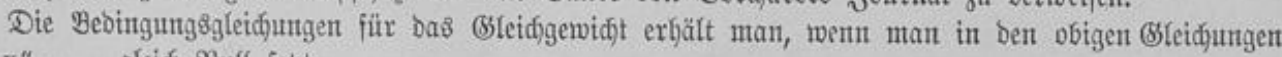
alle $p^{\prime \prime}, x^{\prime \prime}$... gleid) Null jeşt.

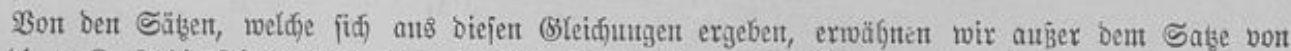

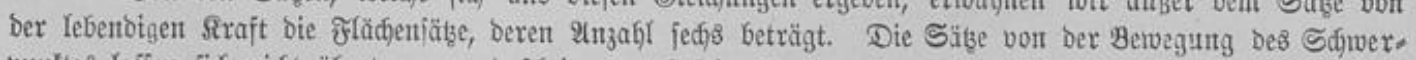

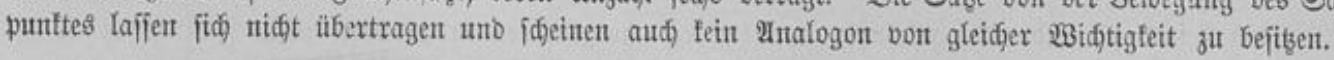

$$
\S 4 .
$$

\section{Bewegung eiues fẹtten Rörpers.}

Die untenblidje fleine Betwegung eines Syjtemts von ftarr mit einantor verbunbenten Bunften ijt

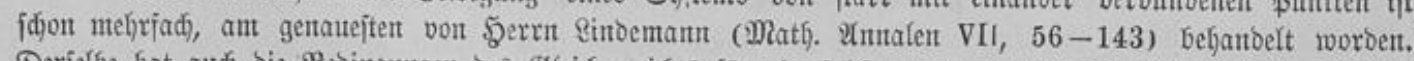

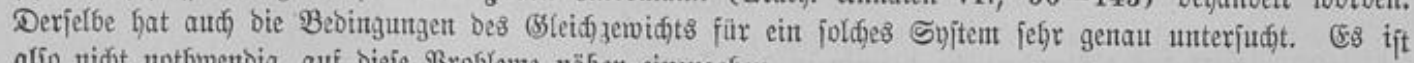
aljo nidjt nothwentig, auf bieje ßrobleme näber einjugefjent.

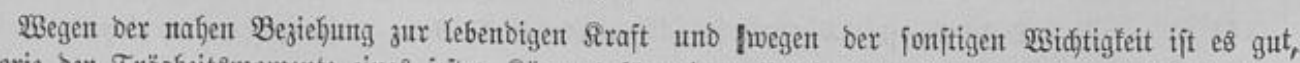

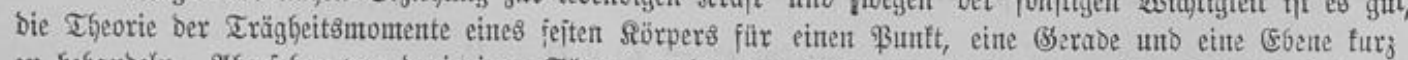

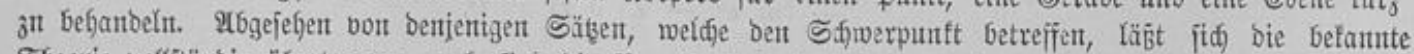

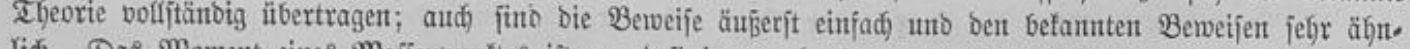
lic.. Das Moment eines Maijempunttes ijt ju befinteren als bas mit $\mathrm{k}^{2}$ multiplijierte \$robuft aus ber 


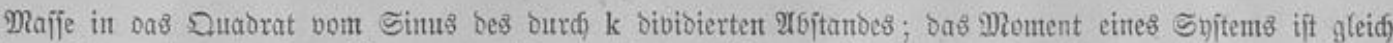

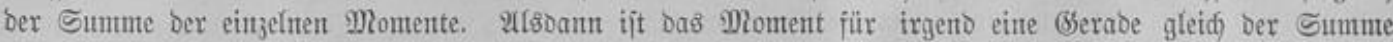
ber Montente für itgento jwei auf einantoer jenfredfte (Ebenten welde fidf in ber (Beraben fdnteiben. Ebenjo ift bie Summe ber Dlontente für brei zu einantoer fentreffte Ebenten gleidh bent in Bejug auf ifren Sdgntitt, punft geltentent Mintente. Enolich iit in ben enolidgen Faumformen bie Sinme ber Mtomente für vier aufo

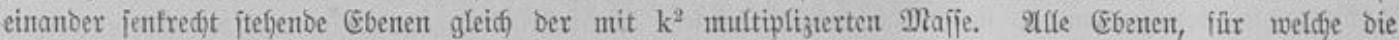
Trägheitsmomente gleif) jint, beriibren basielbe Ellipjoio; alle jo bejtimmten Ellipioide jinto fortiofal. Sie

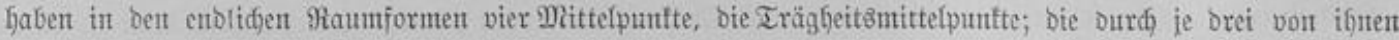

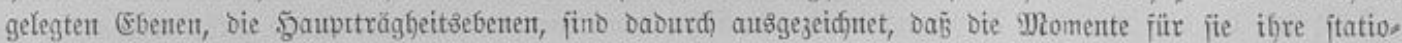

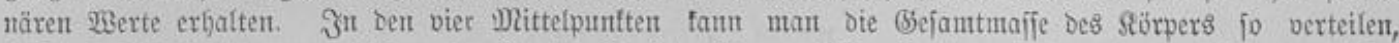

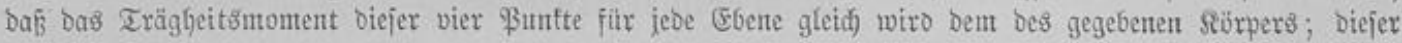
Satz läß̆t eitte bebeutento Erweiterung ju

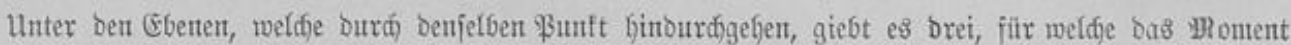

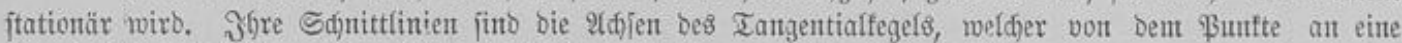

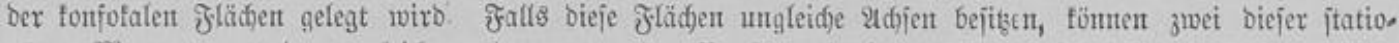

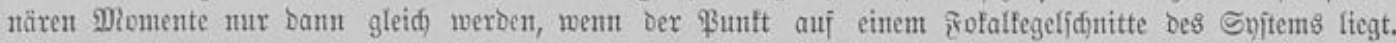

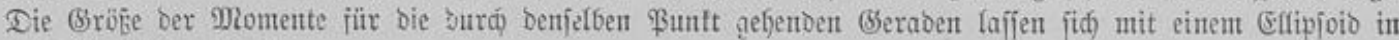
nabe Bezichung jetzen.

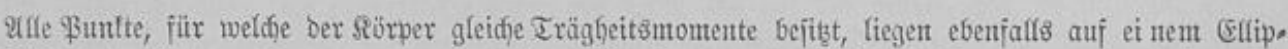

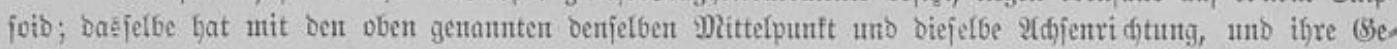
famtheit bilbet ein Syjtem äbnlidjer zläđhen.

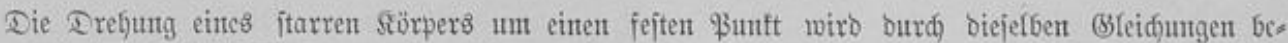

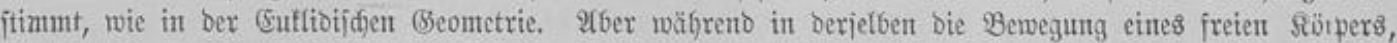

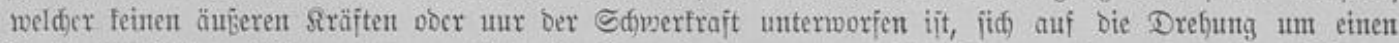
Funft zurïđfjübren läß̄t, verlangen dieje \$robleme in ben antern Raumformen eine eigene Unterjuduntg.

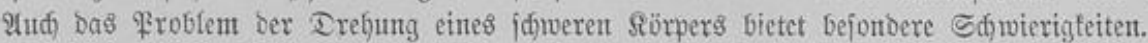

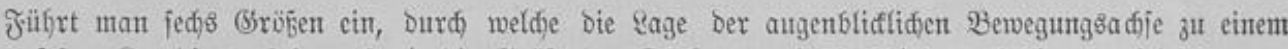

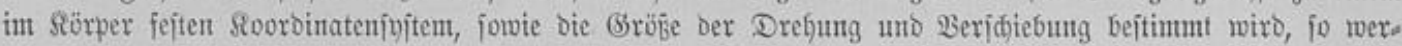

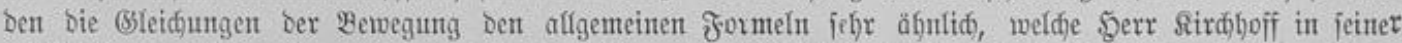

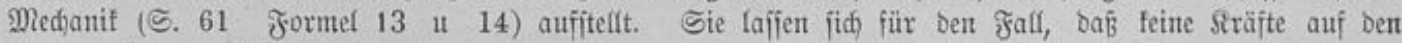

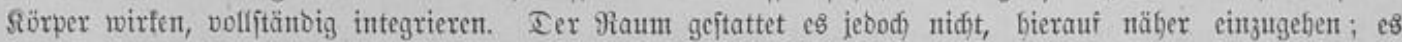

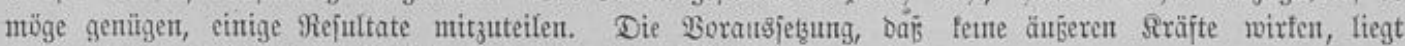

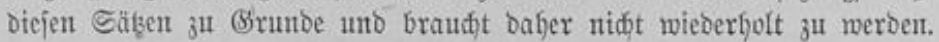

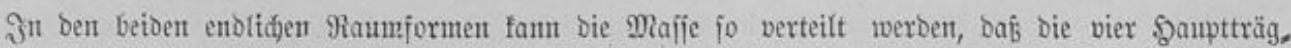

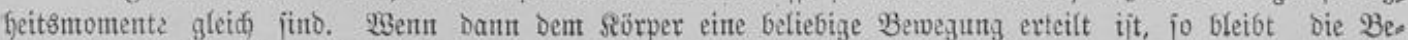

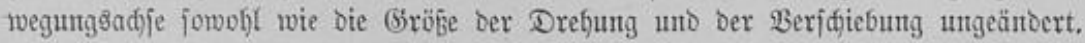

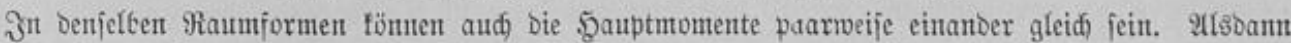

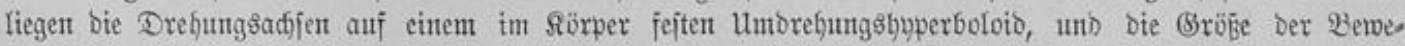
gung ijt unberänberlich)

Wenu Drei 5auptmomente gleiđ) f̈uto, io zerlege man bie augenbliffidje Bewegung auf jolde Weije

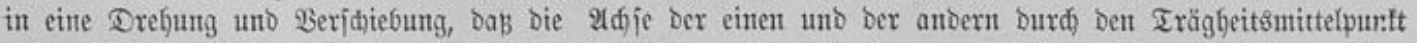

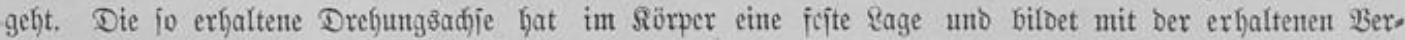

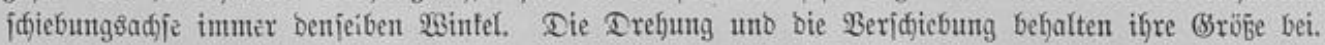

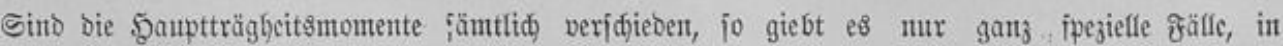




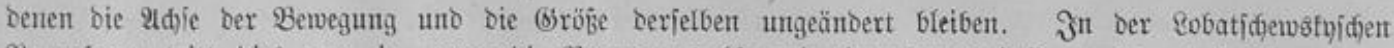

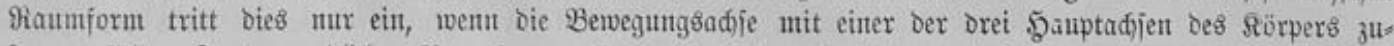

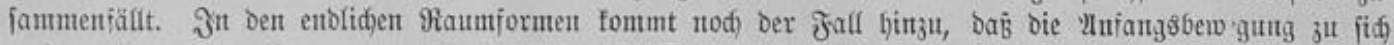

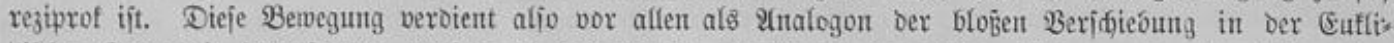

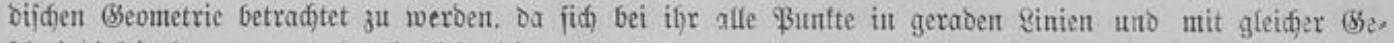

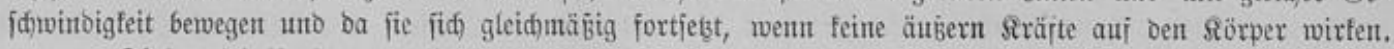

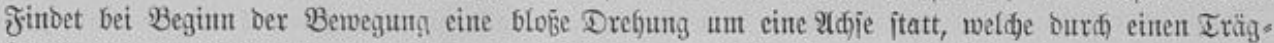
beitsmittelpuntt geht, jo bleibt Die benegung eine Drefjung um biejen \$untt. Das \$roblent fann in biejem Ealle unto bei ber rejiprofen Bewegung ourd) elliptijd)e Funttionen in befanter weife gelöit weroen. 